\title{
Análisis filogenético molecular: Diseño e implementación de algoritmos escalables y fiables y verificación automática de propiedades de una filogenia
}

\author{
J. Álvarez-Jarreta, G. de Miguel, E. Mayordomo \\ Grupo de Ingeniería de Sistemas de Eventos Discretos (GISED) \\ Instituto de Investigación en Ingeniería de Aragón (I3A) \\ Universidad de Zaragoza, Mariano Esquillor s/n, 50018, Zaragoza, Spain. \\ Tel. +34-976762707, Fax +34-976762043, e-mail: jorgeal@unizar.es
}

\begin{abstract}
La filogenética es la ciencia que estudia las relaciones entre organismos basándose en su proximidad evolutiva. La forma más visual y conveniente de representar estas relaciones es a través de los árboles filogenéticos. El análisis filogenético es un proceso formado por distintas etapas cuya finalidad es poder reconstruir dichos árboles. Estas etapas pueden incluir: estudio de modelos evolutivos (modelos matemáticos que intentan explicar de la forma más fiel posible la evolución real de los organismos), análisis estadístico, alineamiento de secuencias,... Actualmente el coste computacional limita de forma práctica tanto la realización de filogenias extensivas (tratando miles y decenas de miles de secuencias) como la aplicación de modelos evolutivos más generales, interesantes y explicativos que el modelo uniforme (usado en tamaños de problema reducidos). Por otro lado, los procesos de secuenciación de cadenas biológicas no están exentos de errores, los cuales pueden aparecer en cualquier lugar de la secuencia. Hasta la fecha, todo proceso de verificación requiere la actuación manual de un experto en el campo, lo que es un proceso muy costoso. El trabajo aborda tres aspectos específicos: i) el desarrollo e implementación de un sistema de inferencia filogenética que concentra varios métodos sobre análisis de secuencias y estudio de filogenias que no se habían unido hasta el momento; ii) el desarrollo e implementación de una aplicación para la detección automática de errores en las cadenas obtenidas en los procesos de secuenciación; iii) el estudio teórico de nuevos algoritmos para la caracterización de problemas entre aquellos que se consideran como no resolubles en la actualidad.
\end{abstract}

